\title{
Color dressed unitarity and recursion for Yang-Mills two-loop all-plus amplitudes
}

\author{
David C. Dunbar, John H. Godwin, Warren B. Perkins $\odot$, and Joseph M. W. Strong \\ College of Science, Swansea University, Swansea SA2 8PP, United Kingdom
}

(Received 18 November 2019; published 13 January 2020)

\begin{abstract}
We present a direct computation of the full color two-loop five-point all-plus Yang-Mills amplitude using four dimensional unitarity and recursion. We present the $S U\left(N_{c}\right)$ amplitudes in compact analytic forms. Our results match the explicit expressions previously computed but do not require full two-loop integral methods.
\end{abstract}

DOI: 10.1103/PhysRevD.101.016009

\section{INTRODUCTION}

Computing perturbative scattering amplitudes is not only a key tool in confronting theories of particle physics with experimental results but is also a gateway for exploring the symmetries and properties of theories which are not always manifest in a Lagrangian approach. Since the standard model of particle physics and many of its potential extensions are gauge theories, gauge theory amplitudes are of particular interest. Within a Yang-Mills gauge theory an $n$-gluon amplitude in may be expanded in the gauge coupling constant,

$$
\mathcal{A}_{n}=g^{n-2} \sum_{\ell \geq 0} a^{\ell} \mathcal{A}_{n}^{(\ell)}
$$

where $a=g^{2} e^{-\gamma_{E} \epsilon} /(4 \pi)^{2-\epsilon}$. Each loop amplitude can be further expanded in terms of color structures, $C^{\lambda}$,

$$
\mathcal{A}_{n}^{(\ell)}=\sum_{\lambda} A_{n: \lambda}^{(\ell)} C^{\lambda}
$$

separating the color and kinematics of the amplitude. The color structures $C^{\lambda}$ may be organized in terms of powers of $N_{c}$.

A great deal of progress has been made in computing $\mathcal{A}_{n}^{(\ell)}$ for tree amplitudes $(\ell=0)$ and one-loop amplitudes $(\ell=1)$ in $S U\left(N_{c}\right)$ gauge theory. However progress in twoloop amplitudes has been more modest: the four gluon amplitude has been computed [1,2] for the full color and helicity structure and there is currently tremendous progress in the computation of the five-point amplitude.

Published by the American Physical Society under the terms of the Creative Commons Attribution 4.0 International license. Further distribution of this work must maintain attribution to the author(s) and the published article's title, journal citation, and DOI. Funded by SCOAP ${ }^{3}$.
The first amplitude to be computed at five point was the leading in color part of the amplitude with all positive helicity external gluons (the all-plus amplitude) which was computed using $d$-dimensional unitarity methods $[3,4]$ and was subsequently presented in a very elegant and compact form [5]. In Ref. [6], it was shown how four-dimensional unitarity techniques could be used to regenerate the fivepoint leading in color amplitude and in Refs. $[7,8]$ the leading in color all-plus amplitudes were obtained for six and seven points, these being the first six- and seven-point amplitudes to be obtained at two-loops. The leading in color five-point amplitudes have been computed for the remaining helicities $[9,10]$. Full color amplitudes are significantly more complicated requiring a larger class of master integrals incorporating nonplanar integrals $[11,12]$. In Ref. [13] the first full color five-point amplitude was presented in QCD.

In this article we will examine the one- and two-loop partial amplitudes using a $U\left(N_{c}\right)$ color trace basis where the fundamental objects are traces of color matrices $T^{a}$ rather than contractions of the structure constants $f^{a b c}$. We examine the particular scattering amplitude in pure gauge theory where the external gluons have identical helicity, $\mathcal{A}_{n}\left(1^{+}, \ldots n^{+}\right)$. This amplitude is fully crossing symmetric which makes computation relatively more tractable but nonetheless is a valuable laboratory for studying the properties of gluon scattering. The all-plus amplitude has a singular structure which is known from general theorems together with a finite remainder part. We present a form for the finite part which is a simple combination of dilogarithms together with rational terms. Specifically we compute directly all the color trace structures for the five-point allplus two-loop amplitude. Our results are in complete agreement with the results recently computed by Badger et al. [13] and are consistent with constraints imposed by group theoretical arguments [14,15].

Our methodology involves computing the polylogarithmic and rational parts of the finite remainder by a 
combination of techniques. The polylogarithms are computed using four-dimensional unitarity cuts and the rational parts are determined by recursion. We use augmented recursion [16] to overcome the issues associated with the presence of double poles.

\section{ONE-LOOP SUBLEADING AMPLITUDES}

An $n$-point tree amplitude can be expanded in a color trace basis as

$$
\mathcal{A}_{n}^{(0)}(1,2,3, \ldots, n)=\sum_{S_{n} / Z_{n}} \operatorname{Tr}\left[T^{a_{1}} \ldots T^{a_{n}}\right] A_{n: 1}^{(0)}\left(a_{1}, a_{2}, \ldots a_{n}\right) .
$$

This separates the color and kinematic structures. The partial amplitudes $A_{n: 1}^{(0)}\left(a_{1}, a_{2}, \ldots a_{n}\right)$ are cyclically symmetric but not fully crossing symmetric. The sum over permutations is over permutations of $(1,2, \ldots n)$ up to this cyclic symmetry. This is not the only expansion and in fact other expansions exist [17] which may be more efficient for some purposes. This color decomposition is valid for both $U\left(N_{c}\right)$ and $S U\left(N_{c}\right)$ gauge theories. If any of the external particles in the $U\left(N_{c}\right)$ case are $U(1)$ particles then the amplitude must vanish. This imposes decoupling identities among the partial amplitudes [18]. For example at tree-level setting leg 1 to be $U(1)$ and extracting the coefficient of $\operatorname{Tr}\left[T^{2} T^{3} \ldots T^{n}\right]$ implies that

$$
\begin{aligned}
& A_{n: 1}^{(0)}(1,2,3, \ldots n)+A_{n: 1}^{(0)}(2,1,3, \ldots n) \\
& \quad+\cdots A_{n: 1}^{(0)}(2, \ldots, 1, n)=0 .
\end{aligned}
$$

This provides a consistency check on the partial amplitudes. At loop level these decoupling identities provide powerful relationships between the different pieces of the amplitude.

In a $U\left(N_{c}\right)$ gauge theory the one-loop $n$-point amplitude can be expanded as [18]

$$
\begin{aligned}
\mathcal{A}_{n}^{(1)}(1,2,3, \ldots, n)= & \sum_{S_{n} / Z_{n}} N_{c} \operatorname{Tr}\left[T^{a_{1}} \ldots T^{a_{n}}\right] A_{n: 1}^{(1)}\left(a_{1}, a_{2}, \ldots a_{n}\right) \\
& +\sum_{r=2}^{[n / 2]+1} \sum_{S_{n} /\left(Z_{r-1} \times Z_{n+1-r}\right)} \operatorname{Tr}\left[T^{a_{1}} \ldots T^{a_{r-1}}\right] \operatorname{Tr}\left[T^{b_{r}} \ldots T^{b_{n}}\right] A_{n: r}^{(1)}\left(a_{1} \ldots a_{r-1} ; b_{r} \ldots b_{n}\right) .
\end{aligned}
$$

The $A_{n: 2}^{(1)}$ are absent (or zero) in the $S U\left(N_{c}\right)$ case. For $n$ even and $r=n / 2+1$ there is an extra $Z_{2}$ in the summation to ensure each color structure only appears once. The partial amplitudes $A_{n: r}^{(1)}\left(a_{1} \ldots a_{r-1} ; b_{r} \ldots b_{n}\right)$ are cyclically symmetric in the sets $\left\{a_{1} \ldots a_{r-1}\right\}$ and $\left\{b_{r} \ldots b_{n}\right\}$ and obey a "flip" symmetry,

$$
\begin{aligned}
& A_{n: r}^{(1)}(1,2, \ldots(r-1) ; r, \ldots n) \\
& \quad=(-1)^{n} A_{n: r}^{(1)}(r-1, \ldots 2,1 ; n, \ldots r) .
\end{aligned}
$$

Amplitudes involving the scattering of gauge bosons also occur in string theories. From a string theory viewpoint the $A_{n: r}^{(1)}$ with $r>1$ would be considered nonplanar contributions arising from attaching gauge bosons to the two edges of a one-loop surface.

Decoupling identities impose relationships among the partial amplitudes. For example setting leg 1 to be $U(1)$ and extracting the coefficient of $\operatorname{Tr}\left[T^{2} T^{3} \ldots T^{n}\right]$ implies

$$
\begin{aligned}
& A_{n: 2}^{(1)}(1 ; 2,3, \ldots n)+A_{n: 1}^{(1)}(1,2,3, \ldots n) \\
& \quad+A_{n: 1}^{(1)}(2,1,3, \ldots n)+\cdots A_{n: 1}^{(1)}(2, \ldots, 1, n)=0
\end{aligned}
$$

and consequently the $A_{n: 2}^{(1)}$ can be expressed as a sum of $(n-1)$ of the $A_{n: 1}^{(1)}$. By repeated application of the decoupling identities all the $A_{n: r}^{(1)}$ can be expressed as sums over the $A_{n: 1}^{(1)}[18]$,

$$
\begin{gathered}
A_{n ; r}^{(1)}(1,2, \ldots, r-1 ; r, r+1, \ldots, n) \\
\quad=(-1)^{r-1} \sum_{\sigma \in C O P\{\alpha\}\{\beta\}} A_{n ; 1}^{(1)}(\sigma)
\end{gathered}
$$

where $\alpha_{i} \in\{\alpha\} \equiv\{r-1, r-2, \ldots, 2,1\}$ and $\beta_{i} \in\{\beta\} \equiv$ $\{r, r+1, \ldots, n-1, n\}$ [Note that the ordering of the first set of indices is reversed with respect to the second]. $\operatorname{COP}\{\alpha\}\{\beta\}$ is the set of all permutations of $\{1,2, \ldots, n\}$ with $n$ held fixed that preserve the cyclic ordering of the $\alpha_{i}$ within $\{\alpha\}$ and of the $\beta_{i}$ within $\{\beta\}$, while allowing for all possible relative orderings of the $\alpha_{i}$ with respect to the $\beta_{i}$. For example if $\{\alpha\}=\{2,1\}$ and $\{\beta\}=\{3,4,5\}$, then $\operatorname{COP}\{\alpha\}\{\beta\}$ contains the twelve elements 

$(2,1,3,4,5)$,
$(2,3,1,4,5)$,
$(2,3,4,1,5)$,
$(3,2,1,4,5)$,
$(3,2,4,1,5)$,
$(3,4,2,1,5)$,
$(1,2,3,4,5)$,
$(1,3,2,4,5)$,
$(1,3,4,2,5)$,
$(3,1,2,4,5)$,
$(3,1,4,2,5)$,
$(3,4,1,2,5)$.

The simplest one-loop QCD $n$-gluon helicity amplitude is the all-plus amplitude with all external helicities positive. The tree amplitude vanishes for this particular amplitude and, consequently, the one-loop amplitude is rational (to order $\epsilon^{0}$ ). The leading in color one-loop partial amplitude has an all- $n$ expression [19] $]^{1}$

$$
A_{n: 1}^{(1)}\left(1^{+}, 2^{+}, \ldots, n^{+}\right)=-\frac{i}{3} \frac{1}{\langle 12\rangle\langle 23\rangle \ldots\langle n 1\rangle} \sum_{1 \leq i<j<k<l \leq n} \operatorname{tr}_{-}[i j k l]+O(\epsilon) .
$$

This expression is order $\epsilon^{0}$ but all- $\epsilon$ expressions exist for the first few amplitudes in this series [20]. The subleading terms can be obtained from decoupling identities. We have obtained compact expressions, to order $\epsilon^{0}$, for these:

$$
\begin{aligned}
& A_{n: 1}^{(1)}\left(1^{+}, 2^{+}, 3^{+}, \ldots, n^{+}\right)=-\frac{i}{3} \frac{1}{\langle 12\rangle\langle 23\rangle \ldots\langle n 1\rangle} \sum_{1 \leq i<j<k<l \leq n} \operatorname{tr}_{-}[i j k l], \\
& A_{n: 2}^{(1)}\left(1^{+} ; 2^{+}, 3^{+}, \ldots, n^{+}\right)=-i \frac{1}{\langle 23\rangle\langle 34\rangle \ldots\langle n 2\rangle} \sum_{2 \leq i<j \leq n}[1 i]\langle i j\rangle[j 1],
\end{aligned}
$$

and for $r \geq 3$

$$
A_{n: r}^{(1)}\left(1^{+}, 2^{+}, \ldots, r-1^{+} ; r^{+} \ldots n^{+}\right)=-2 i \frac{\left(K_{1 \ldots r-1}^{2}\right)^{2}}{(\langle 12\rangle\langle 23\rangle \ldots\langle(r-1) 1\rangle)(\langle r(r+1)\rangle \ldots\langle n r\rangle)}
$$

These expressions are remarkably simple given the number of terms arising in the naive application of Eq. (2.6): the number of terms in the numerator of a single $A_{n: 1}^{(1)}$ grows as

$$
\frac{1}{24} n(n-1)(n-2)(n-3) \text {, }
$$

while the summation over $C O P$ terms grows with $n$ as

$$
\sim \frac{(n-1) !}{(r-2) !(n-r) !} .
$$

A further complication arises for one-loop amplitudes where the external helicities are not identical, the simplest case being the single-minus amplitude with one negative helicity gluon and the rest positive helicity. Double poles arise in these amplitudes for complex momenta where factorizations as in Fig. 1 occur.

The factorization takes the form

$$
\begin{gathered}
\frac{V^{(1)}\left(a^{+}, b^{+}, K^{+}\right)}{s_{a b}} \times \frac{1}{s_{a b}} \times A_{n-1: 1}^{(0)}\left(K^{-}, \ldots\right) \\
\sim \frac{[a b]}{\langle a b\rangle^{2}} \times A_{n-1: 1}^{(0)}\left(K^{-}, \ldots\right)
\end{gathered}
$$

\footnotetext{
${ }^{1}$ Here a null momentum is represented as a pair of two component spinors $p^{\mu}=\sigma_{\alpha \dot{\alpha}}^{\mu} \lambda^{\alpha} \bar{\lambda}^{\alpha}$. We are using a spinor helicity formalism with the usual spinor products $\langle a b\rangle=\epsilon_{\alpha \beta} \lambda_{a}^{\alpha} \lambda_{b}^{\beta}$ and $[a b]=-\epsilon_{\dot{\alpha} \dot{\beta}} \bar{\lambda}_{a} \bar{\lambda}^{\dot{\alpha}} \bar{\lambda}_{b}^{\dot{\beta}}$. Also $\quad s_{a b}=\left(k_{a}+k_{b}\right)^{2}=\langle a b\rangle[b a]=$ $\langle a|b| a], \operatorname{tr}_{-}[i j k l] \equiv \operatorname{tr}\left(\frac{\left(1-\gamma_{5}\right)}{2} \not k_{i} k_{j} k_{k} k_{l}\right)=\langle i j\rangle[j k]\langle k l\rangle[l i], \operatorname{tr}_{+}[i j k l] \equiv$ $\operatorname{tr}\left(\frac{\left(1+\gamma_{5}\right)}{2} \not k_{i} \not k_{j} \not k_{k} \not k_{l}\right)=[i j]\langle j k\rangle[k l]\langle l i\rangle$ and $\epsilon(i, j, k, l)=\operatorname{tr}_{+}[i j k l]-$ $\operatorname{tr}_{-}[i j k l]$.
}

where

$$
\frac{V^{(1)}\left(a^{+}, b^{+}, K^{+}\right)}{s_{a b}}=-\frac{i}{3} \frac{[a b][b K][K a]}{s_{a b}}
$$

is the one-loop three-point vertex [21]. For $n>4$, the allplus one-loop amplitude does not contain double poles since the tree amplitude on the right-hand side of Fig. 1 vanishes. The double poles in the single-minus amplitudes can be seen explicitly in the five-point case [22],

$$
\begin{aligned}
& A_{5: 1}^{(1)}\left(1^{-}, 2^{+}, 3^{+}, 4^{+}, 5^{+}\right) \\
& =\frac{i}{3} \frac{1}{\langle 34\rangle^{2}}\left[-\frac{[25]^{3}}{[12][51]}+\frac{\langle 14\rangle^{3}[45]\langle 35\rangle}{\langle 12\rangle\langle 23\rangle\langle 45\rangle^{2}}-\frac{\langle 13\rangle^{3}[32]\langle 42\rangle}{\langle 15\rangle\langle 54\rangle\langle 32\rangle^{2}}\right]
\end{aligned}
$$



FIG. 1. The origin of the double pole. The double pole corresponds to the coincidence of the singularity arising in the 3-pt integral with the factorisation corresponding to $K^{2}=s_{a b} \rightarrow 0$. 
where there are $\langle a b\rangle^{-2}$ singularities for $\langle a b\rangle=\langle 23\rangle,\langle 34\rangle$ and $\langle 45\rangle$.

Again the subleading in color partial amplitudes can be obtained in terms of the leading in color partial amplitudes using decoupling identities. The naive application of Eq. (2.6) obscures the simplicity of the subleading terms. In particular, there are no double poles in the one-loop subleading partial amplitudes for $n>4$.

To demonstrate this we first consider the partial amplitude $A_{n: 2}^{(1)}\left(a_{1} ; b_{2}, b_{3}, b_{4}, \ldots b_{n}\right)$. This can be expressed as a sum over the $A_{n: 1}^{(1)}$,

$$
\begin{aligned}
A_{n: 2}^{(1)}\left(a_{1} ; b_{2}, b_{3}, \ldots b_{n}\right)= & -A_{n: 1}^{(1)}\left(a_{1}, b_{2}, b_{3}, \ldots b_{n}\right) \\
& -A_{n: 1}^{(1)}\left(b_{2}, a_{1}, b_{3}, \ldots b_{n}\right) \ldots \\
& -A_{n: 1}^{(1)}\left(b_{2}, b_{3}, \ldots a_{1}, b_{n}\right)
\end{aligned}
$$

where the sum is over the $n-1$ distinct places where $a_{1}$ may be inserted within $b_{2}, b_{3} \ldots b_{n}$. If we consider the double pole in $\left\langle a_{1} b_{2}\right\rangle$ this will only occur in the first two terms and will be of the form

$$
\begin{aligned}
- & \frac{V^{(1)}\left(a_{1}^{+}, b_{2}^{+}, K^{+}\right)}{s_{a_{1} b_{2}}^{2}} \times A_{n-1: 1}^{(0)}\left(K^{-}, b_{3}, \ldots b_{n}\right) \\
& -\frac{V^{(1)}\left(b_{2}^{+}, a_{1}^{+}, K^{+}\right)}{s_{a_{1} b_{2}}^{2}} \times A_{n-1: 1}^{(0)}\left(K^{-}, b_{3}, \ldots b_{n}\right),
\end{aligned}
$$

which vanishes since $V^{(1)}\left(a^{+}, b^{+}, K^{+}\right)$is antisymmetric. The double pole in $\left\langle b_{2} b_{3}\right\rangle$ also vanishes, but via a different route. Only the second term in Eq. (2.15) does not contribute and we obtain

$$
\begin{aligned}
- & \frac{V^{(1)}\left(b_{2}^{+}, b_{3}^{+}, K^{+}\right)}{s_{b_{2} b_{3}}^{2}} \times\left(A_{n-1: 1}^{(0)}\left(a_{1}, K^{-}, b_{4}, \ldots b_{n}\right)\right. \\
& +A_{n-1: 1}^{(0)}\left(K^{-}, a_{1}, b_{4}, \ldots b_{n}\right)+\cdots \\
& \left.+A_{n-1: 1}^{(0)}\left(K^{-}, b_{4}, \ldots a_{1}, b_{n}\right)\right) .
\end{aligned}
$$

This vanishes due to the decoupling identity for the tree amplitude $A_{n-1: 1}^{(0)}$ (2.2). Similar arguments show the vanishing of double poles for all $A_{n: r}^{(1)}$ with $r>1$.

The simplifications in the subleading terms allow us to present some compact $n$-point expressions. Explicitly, we can find all- $n$ formulas for $A_{n: 2}^{(1)}\left(1^{-} ; 2^{+}, \ldots n^{+}\right)$and $A_{n: 3}^{(1)}\left(1^{-}, 2^{+} ; 3^{+}, \ldots n^{+}\right)$:

$A_{n: 2}^{(1)}\left(1^{-} ; 2^{+}, 3^{+}, \ldots, n^{+}\right)=\frac{-i \sum_{2 \leq i<j \leq n}\langle 1|i j| 1\rangle}{\langle 23\rangle\langle 34\rangle \ldots\langle(n-1) n\rangle\langle n 2\rangle}$

and

$A_{n: 3}^{(1)}\left(1^{-}, 2^{+} ; 3^{+}, \ldots, n^{+}\right)=\sum_{Z_{(3 \ldots n)}} \frac{i \sum_{2 \leq i<j \leq n}\langle 1|i j| 1\rangle}{\langle 23\rangle\langle 34\rangle \ldots\langle(n-1) n\rangle\langle n 2\rangle}$

where $Z_{(3 \ldots n)}$ is the set of cyclic permutations of the set $(3, \ldots n)$.

The vanishing of the $\left\langle b_{2} b_{3}\right\rangle$ double poles in Eq. (2.17) uses a tree level identity, so we do not expect the argument to extend beyond one-loop. Specifically if we consider $A_{n: 2}^{(2)}\left(a_{1} ; b_{2}, b_{3}, b_{4}, \ldots b_{n}\right)$, a formula for the double pole in $\left\langle b_{2} b_{3}\right\rangle$ akin to Eq. (2.17) will exist but with the tree amplitudes $A_{n-1: 1}^{(0)}$ replaced by their one-loop equivalents $A_{n-1: 1}^{(1)}$. The combination of $A_{n-1: 1}^{(1)}$ is that of the decoupling identity (2.5) so the double pole does not vanish but instead is proportional to

$$
\frac{V^{(1)}\left(b_{2}^{+}, b_{3}^{+}, K^{+}\right)}{s_{b_{2} b_{3}}^{2}} \times A_{n-1: 2}^{(1)}\left(a_{1} ; K^{-}, b_{4}, \ldots b_{n}\right) .
$$

\section{TWO-LOOP AMPLITUDES}

A general two-loop amplitude may be expanded in a color trace basis as

$$
\begin{aligned}
\mathcal{A}_{n}^{(2)}(1,2, \ldots, n)= & N_{c}^{2} \sum_{S_{n} / Z_{n}} \operatorname{Tr}\left(T^{a_{1}} T^{a_{2}} \ldots T^{a_{n}}\right) A_{n: 1}^{(2)}\left(a_{1}, a_{2}, \ldots, a_{n}\right) \\
& +N_{c} \sum_{r=2}^{[n / 2]+1} \sum_{S_{n} /\left(Z_{r-1} \times Z_{n+1-r}\right)} \operatorname{Tr}\left(T^{a_{1}} T^{a_{2}} \ldots T^{a_{r-1}}\right) \operatorname{Tr}\left(T^{b_{r}} \ldots T^{b_{n}}\right) A_{n: r}^{(2)}\left(a_{1}, a_{2}, \ldots, a_{r-1} ; b_{r} \ldots b_{n}\right) \\
& +\sum_{s=1}^{[n / 3]} \sum_{t=s}^{[(n-s) / 2]} \sum_{S_{n} /\left(Z_{s} \times Z_{t} \times Z_{n-s-t}\right)} \operatorname{Tr}\left(T^{a_{1}} \ldots T^{a_{s}}\right) \operatorname{Tr}\left(T^{b_{s+1}} \ldots T^{b_{s+t}}\right) \operatorname{Tr}\left(T^{c_{s+t+1}} \ldots T^{c_{n}}\right) \\
& \times A_{n: s, t}^{(2)}\left(a_{1}, \ldots, a_{s} ; b_{s+1} \ldots b_{s+t} ; c_{s+t+1} \ldots c_{n}\right) \\
& +\sum_{S_{n} / Z_{n}} \operatorname{Tr}\left(T^{a_{1}} T^{a_{2}} \ldots T^{a_{n}}\right) A_{n: 1 B}^{(2)}\left(a_{1}, a_{2}, \ldots, a_{n}\right) .
\end{aligned}
$$


Again, for $n$ even and $r=n / 2+1$ there is an extra $Z_{2}$ in the summation to ensure each color structure only appears once. In the $s, t$ summations there is an extra $Z_{2}$ when exactly two of $s, t$ and $n-s-t$ are equal and an extra $S_{3}$ when all three are equal.

For five-point amplitudes this reduces to

$$
\begin{aligned}
\mathcal{A}_{5}^{(2)}(1,2,3,4,5)= & N_{c}^{2} \sum_{S_{5} / Z_{5}} \operatorname{Tr}\left(T^{a_{1}} T^{a_{2}} T^{a_{3}} T^{a_{4}} T^{a_{5}}\right) A_{5: 1}^{(2)}\left(a_{1}, a_{2}, a_{3}, a_{4}, a_{5}\right) \\
& +N_{c} \sum_{S_{5} / Z_{4}} \operatorname{Tr}\left(T^{a_{1}}\right) \operatorname{Tr}\left(T^{b_{2}} T^{b_{3}} T^{b_{4}} T^{b_{5}}\right) A_{5: 2}^{(2)}\left(a_{1} ; b_{2}, b_{3}, b_{4}, b_{5}\right) \\
& +N_{c} \sum_{S_{5} /\left(Z_{2} \times Z_{3}\right)} \operatorname{Tr}\left(T^{a_{1}} T^{a_{2}}\right) \operatorname{Tr}\left(T^{b_{3}} T^{b_{4}} T^{b_{5}}\right) A_{5: 3}^{(2)}\left(a_{1}, a_{2} ; b_{3}, b_{4}, b_{5}\right) \\
& +\sum_{S_{5} /\left(Z_{2} \times Z_{3}\right)} \operatorname{Tr}\left(T^{a_{1}}\right) \operatorname{Tr}\left(T^{b_{2}}\right) \operatorname{Tr}\left(T^{c_{3}} T^{c_{4}} T^{c_{5}}\right) A_{5: 1,1}^{(2)}\left(a_{1} ; b_{2} ; c_{3}, c_{4}, c_{5}\right) \\
& +\sum_{S_{5} /\left(Z_{2} \times Z_{2} \times Z_{2}\right)} \operatorname{Tr}\left(T^{a_{1}}\right) \operatorname{Tr}\left(T^{b_{2}} T^{b_{3}}\right) \operatorname{Tr}\left(T^{c_{4}} T^{c_{5}}\right) A_{5: 1,2}^{(2)}\left(a_{1} ; b_{2}, b_{3} ; c_{4}, c_{5}\right) \\
& +\sum_{S_{5} / Z_{5}} \operatorname{Tr}\left(T^{a_{1}} T^{a_{2}} T^{a_{3}} T^{a_{4}} T^{a_{5}}\right) A_{5: 1 B}^{(2)}\left(a_{1}, a_{2}, a_{3}, a_{4}, a_{5}\right)
\end{aligned}
$$

which for an $S U\left(N_{c}\right)$ gauge group simplifies to

$$
\begin{aligned}
\mathcal{A}_{5}^{(2)}(1,2,3,4,5)= & N_{c}^{2} \sum_{S_{5} / Z_{5}} \operatorname{Tr}\left(T^{a_{1}} T^{a_{2}} T^{a_{3}} T^{a_{4}} T^{a_{5}}\right) A_{5: 1}^{(2)}\left(a_{1}, a_{2}, a_{3}, a_{4}, a_{5}\right) \\
& +N_{c} \sum_{S_{5} /\left(Z_{2} \times Z_{3}\right)} \operatorname{Tr}\left(T^{a_{1}} T^{a_{2}}\right) \operatorname{Tr}\left(T^{b_{3}} T^{b_{4}} T^{b_{5}}\right) A_{5: 3}^{(2)}\left(a_{1}, a_{2} ; b_{3}, b_{4}, b_{5}\right) \\
& +\sum_{S_{5} / Z_{5}} \operatorname{Tr}\left(T^{a_{1}} T^{a_{2}} T^{a_{3}} T^{a_{4}} T^{a_{5}}\right) A_{5: 1 B}^{(2)}\left(a_{1}, a_{2}, a_{3}, a_{4}, a_{5}\right) .
\end{aligned}
$$

Thus there are three independent functions to be determined: $A_{5: 1}^{(2)}, A_{5: 3}^{(2)}$ and $A_{5: 1 B}^{(2)}$. By themselves the $U(1)$ decoupling identities do not determine any of the three, however they can be used to obtain the specifically $U\left(N_{c}\right)$ functions $A_{5: 2}^{(2)}, A_{5: 1,1}^{(2)}$ and $A_{5: 1,2}^{(2)}$ :

$$
\begin{aligned}
A_{5: 2}^{(2)}(1 ; 2,3,4,5) & =-A_{5: 1}^{(2)}(1,2,3,4,5)-A_{5: 1}^{(2)}(2,1,3,4,5)-A_{5: 1}^{(2)}(2,3,1,4,5)-A_{5: 1}^{(2)}(2,3,4,1,5), \\
A_{5: 1,1}^{(2)}(4 ; 5 ; 1,2,3) & =-A_{5: 2}^{(2)}(5 ; 1,2,3,4)-A_{5: 2}^{(2)}(5 ; 1,2,4,3)-A_{5: 2}^{(2)}(5 ; 1,4,2,3)-A_{5: 3}^{(2)}(4,5 ; 1,2,3) \\
& =-A_{5: 3}^{(2)}(4,5 ; 1,2,3)+\sum_{C O P\{4,5\}\{1,2,3\}} A_{5: 1}^{(2)}(1,2,3,4,5)
\end{aligned}
$$

and

$$
A_{5: 1,2}^{(2)}(1 ; 2,3 ; 4,5)=-A_{5: 3}^{(2)}(2,3 ; 1,4,5)-A_{5: 3}^{(2)}(2,3 ; 1,5,4)-A_{5: 3}^{(2)}(4,5 ; 1,2,3)-A_{5: 3}^{(2)}(4,5 ; 1,3,2)=0 .
$$

Decoupling identities do not relate the $A_{n: 1 B}^{(2)}$ to the other terms but do impose a treelike identity,

$$
A_{n: 1 B}^{(2)}(1,2,3, \ldots n)+A_{n: 1 B}^{(2)}(2,1,3, \ldots n)+\cdots A_{n: 1 B}^{(2)}(2, \ldots, 1, n)=0,
$$

which in itself does not specify $A_{n: 1 B}^{(2)}$ completely. There are however further color restrictions beyond the decoupling identities $[14,15]$ which may be obtained by recursive methods. These, together with Eq. (3.5) determine the $A_{5: 1 B}^{(2)}$ in terms of the $A_{5: 1}$ and $A_{5: 3}$ 


$$
\begin{aligned}
A_{5: 1 B}^{(2)}(1,2,3,4,5)= & -A_{5: 1}^{(2)}(1,2,4,3,5)+2 A_{5: 1}^{(2)}(1,2,5,3,4)+A_{5: 1}^{(2)}(1,2,5,4,3)-A_{5: 1}^{(2)}(1,3,2,4,5) \\
& +2 A_{5: 1}^{(2)}(1,3,4,2,5)-5 A_{5: 1}^{(2)}(1,3,5,2,4)-2 A_{5: 1}^{(2)}(1,3,5,4,2)+2 A_{5: 1}^{(2)}(1,4,2,3,5) \\
& +A_{5: 1}^{(2)}(1,4,3,2,5)+2 A_{5: 1}^{(2)}(1,4,5,2,3)+A_{5: 1}^{(2)}(1,4,5,3,2) \\
& -\frac{1}{2} \sum_{Z_{5}(1,2,3,4,5)}\left(A_{5: 3}^{(2)}(1,2 ; 3,4,5)-A_{5: 3}^{(2)}(1,3 ; 2,4,5)\right) .
\end{aligned}
$$

Our calculation we determine $A_{5: 1 B}^{(2)}$ directly and we use Eq. (3.6) as a consistency check.

\section{SINGULARITY STRUCTURE OF THE ALL-PLUS TWO-LOOP AMPLITUDES}

The IR singular structure of a color partial amplitude is determined by general theorems [23]. Consequently we can split the amplitude into singular terms $U_{n: \lambda}^{(2)}$ and finite terms $F_{n: \lambda}^{(2)}$,

$$
A_{n: \lambda}^{(2)}=U_{n: \lambda}^{(2)}+F_{n: \lambda}^{(2)}+\mathcal{O}(\epsilon)
$$

As the all-plus tree amplitude vanishes, $U_{n: \lambda}^{(2)}$ simplifies considerably and is at worst $1 / \epsilon^{2}$. In general an amplitude has UV divergences, collinear IR divergences and soft IR divergences. As the tree amplitude vanishes, both the UV divergences and collinear IR divergences are proportional to $n$ and cancel leaving only the soft IR singular terms [24]. The leading case, $U_{n: 1}^{(2)}$, is proportional to the one-loop amplitude,

$$
U_{n: 1}^{(2)}=A_{n: 1}^{(1)} \times I_{n}^{(2)}
$$

where

$$
I_{n}^{(2)}=\left[-\sum_{i=1}^{n} \frac{1}{\epsilon^{2}}\left(\frac{\mu^{2}}{-s_{i, i+1}}\right)^{\epsilon}\right] .
$$
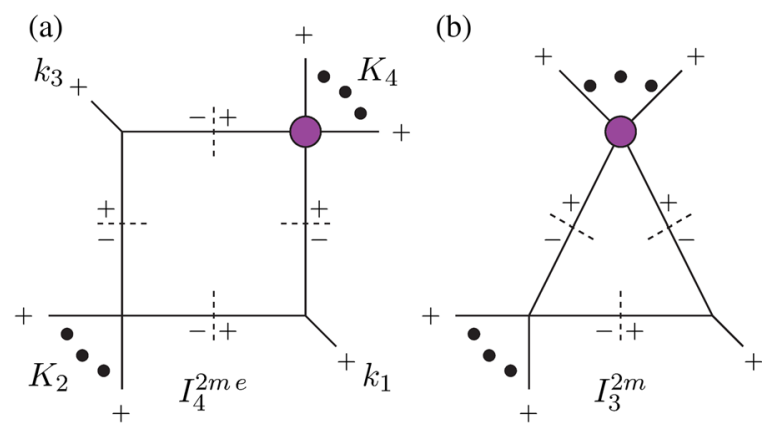

(c)



In Appendix A the form of the two-loop IR divergences for the other unrenormalized partial amplitudes are presented in a color trace basis.

Given the general expressions for $U_{n: \lambda}^{(2)}$, the challenge is to compute the finite parts of the amplitude: $F_{n: \lambda}^{(2)}$. This finite remainder function $F_{n: \lambda}^{(2)}$ can be further split into polylogarithmic and rational pieces,

$$
F_{n: \lambda}^{(2)}=P_{n: \lambda}^{(2)}+R_{n: \lambda}^{(2)}
$$

We calculate the former piece using four-dimensional unitarity and the latter using recursion.

\section{UNITARITY}

$D$-dimensional unitarity techniques can be used to generate the integrands [3] for the five-point amplitude which can then be integrated to obtain the amplitude [5]. However the organization of the amplitude in the previous section allows us to obtain the finite polylogarithms using four-dimensional unitarity $[25,26]$ where the cuts are evaluated in four dimensions with the corresponding simplifications. With this simplification the all-plus oneloop amplitude effectively becomes an additional on-shell vertex and the two-loop cuts effectively become one-loop cuts with a single insertion of this vertex. The nonvanishing four-dimensional cuts are shown in Fig. 2.

The cuts allow us to determine the coefficients, $\alpha_{i}$, of box, triangle and bubble functions in the amplitude. The integral functions are

FIG. 2. Four dimensional cuts of the two-loop all-plus amplitude involving an all-plus one-loop vertex (indicated by a shaded disk). In the boxes $K_{2}$ may be null but $K_{4}$ must contain at least two external legs. 


$$
\begin{gathered}
I_{2}\left(K^{2}\right)=\frac{\left(-K^{2}\right)^{-\epsilon}}{\epsilon(1-2 \epsilon)}, \\
I_{3}^{1 \mathrm{~m}}\left(K^{2}\right)=\frac{1}{\epsilon^{2}}\left(-K^{2}\right)^{-1-\epsilon}, \quad I_{3}^{2 \mathrm{~m}}\left(K_{1}^{2}, K_{2}^{2}\right)=\frac{1}{\epsilon^{2}} \frac{\left(-K_{1}^{2}\right)^{-\epsilon}-\left(-K_{2}^{2}\right)^{-\epsilon}}{\left(-K_{1}^{2}\right)-\left(-K_{2}^{2}\right)},
\end{gathered}
$$

and

$$
\begin{aligned}
I_{4}^{2 \mathrm{me}}\left(S, T, K_{2}^{2}, K_{4}^{2}\right)= & -\frac{2}{S T-K_{2}^{2} K_{4}^{2}}\left[-\frac{1}{\epsilon^{2}}\left[(-S)^{-\epsilon}+(-T)^{-\epsilon}-\left(-K_{2}^{2}\right)^{-\epsilon}-\left(-K_{4}^{2}\right)^{-\epsilon}\right]\right. \\
& +\mathrm{Li}_{2}\left(1-\frac{K_{2}^{2}}{S}\right)+\mathrm{Li}_{2}\left(1-\frac{K_{2}^{2}}{T}\right)+\mathrm{Li}_{2}\left(1-\frac{K_{4}^{2}}{S}\right)+\mathrm{Li}_{2}\left(1-\frac{K_{4}^{2}}{T}\right) \\
& \left.-\mathrm{Li}_{2}\left(1-\frac{K_{2}^{2} K_{4}^{2}}{S T}\right)+\frac{1}{2} \ln ^{2}\left(\frac{S}{T}\right)\right]
\end{aligned}
$$

where $S=\left(k_{1}+K_{2}\right)^{2}$ and $T=\left(k_{1}+K_{4}\right)^{2}$.

The bubbles in principle would determine the $(-s)^{\epsilon} / \epsilon$ infinities. However, explicit calculation using, for example, a canonical basis approach [27] shows that they have zero coefficient. This is a property of this particular helicity configuration and is due to the vanishing of the tree amplitude. The triangles only contribute to $U_{n: \lambda}^{(2)}$, while the box functions contribute to both $U_{n: \lambda}^{(2)}$ and the finite polylogarithms. Separating these pieces we have

$I_{4}^{2 m e}\left(S, T, K_{2}^{2}, K_{4}^{2}\right)=\left.I_{4}^{2 m e}\right|_{\mathrm{IR}}-\frac{2}{S T-K_{2}^{2} K_{4}^{2}} \mathrm{~F}^{2 m}\left[S, T, K_{2}^{2}, K_{4}^{2}\right]$

where $F^{2 m}$ is a dimensionless combination of polylogarithms.

The IR terms combine to give the correct IR singularities [28],

$$
\begin{aligned}
& \left(\left.\sum \alpha_{i} I_{4, i}^{2 \mathrm{me}}\right|_{\mathrm{IR}}+\sum \alpha_{i} I_{3, i}^{2 \mathrm{~m}}+\sum \alpha_{i} I_{3, i}^{1 \mathrm{~m}}\right)_{\lambda} \\
& =U_{n: \lambda}^{(1), \epsilon^{0}}\left(1^{+}, 2^{+}, \ldots, n^{+}\right)
\end{aligned}
$$

where $U_{n}^{(2), \epsilon^{0}}\left(1^{+}, 2^{+}, \ldots, n^{+}\right)$is the order $\epsilon^{0}$ truncation. We have checked the relation of (5.5) by using four dimensional unitarity techniques to compute the coefficients and then comparing to the expected form of $U_{n}^{(2)}$ given in Appendix A for $n$ up to 9 points.

The remaining parts of the box integral functions generate the finite polylogarithms. The expression for $P_{n}^{(2)}$ is [28]

$$
P_{n}^{(2)}=\sum_{i} c_{i} \mathrm{~F}_{i}^{2 m}
$$

where

$$
\begin{aligned}
\mathrm{F}^{2 \mathrm{~m}}\left[S, T, K_{2}^{2}, K_{4}^{2}\right]= & \mathrm{Li}_{2}\left(1-\frac{K_{2}^{2}}{S}\right)+\mathrm{Li}_{2}\left(1-\frac{K_{2}^{2}}{T}\right) \\
& +\mathrm{Li}_{2}\left(1-\frac{K_{4}^{2}}{S}\right)+\mathrm{Li}_{2}\left(1-\frac{K_{4}^{2}}{T}\right) \\
& -\mathrm{Li}_{2}\left(1-\frac{K_{2}^{2} K_{4}^{2}}{S T}\right)+\frac{1}{2} \ln ^{2}\left(\frac{S}{T}\right)
\end{aligned}
$$

and, in the specific case where $K_{2}^{2}=0$,

$$
\begin{aligned}
\mathrm{F}^{1 \mathrm{~m}}\left[S, T, K_{4}^{2}\right] \equiv & \mathrm{F}^{2 \mathrm{~m}}\left[S, T, 0, K_{4}^{2}\right] \\
= & \mathrm{Li}_{2}\left(1-\frac{K_{4}^{2}}{S}\right)+\mathrm{Li}_{2}\left(1-\frac{K_{4}^{2}}{T}\right) \\
& +\frac{1}{2} \ln ^{2}\left(\frac{S}{T}\right)+\frac{\pi^{2}}{6} .
\end{aligned}
$$

Let us now consider the specific five-point case where only the $K_{2}^{2}=0$ case occurs.

In this case the one-loop corner is a four-point amplitude and the color partial amplitudes simplify since

$A_{4: 1}^{(1)}(1,2,3,4)=A_{4: 1}^{(1)}(1,2,4,3)=A_{4: 1}^{(1)}(1,3,2,4)$,

which implies that

$$
\begin{aligned}
A_{4: 2}^{(1)}(1 ; 2,3,4) & =-3 A_{4: 1}^{(1)}(1,2,3,4) \\
\text { and } \quad A_{4: 3}^{(1)}(1,2 ; 3,4) & =6 A_{4: 1}^{(1)}(1,2,3,4)
\end{aligned}
$$

so that the full color amplitude factorizes into color and kinematic terms 


$$
\mathcal{A}_{4}^{(1)}\left(\ell_{1}, \ell_{4}, d, e\right)=\mathcal{C} \times A_{4: 1}^{(1)}\left(\ell_{4}, d, e, \ell_{1}\right)
$$

where the momentum labels are specified in Fig. 3.

Since the three-point tree amplitudes also factorize, the quadruple cut of this box function factorizes as

$$
\begin{aligned}
\mathcal{C}^{\prime} & \times A_{3}^{(0)}\left(a, \ell_{2}, \ell_{1}\right) A_{3}^{(0)}\left(b, \ell_{3}, \ell_{2}\right) A_{3}^{(0)}\left(c, \ell_{4}, \ell_{3}\right) \\
& \times A_{4: 1}^{(1)}\left(\ell_{4}, d, e, \ell_{1}\right) .
\end{aligned}
$$

The solution to the quadruple cuts in this case is

$$
\begin{aligned}
& \ell_{2}=-\frac{\langle b a\rangle}{\langle c a\rangle} \bar{\lambda}_{b} \lambda_{c}, \quad \ell_{3}=-\frac{\langle b c\rangle}{\langle a c\rangle} \bar{\lambda}_{b} \lambda_{a}, \\
& \ell_{1}=\frac{\langle a c\rangle \bar{\lambda}_{a}+\langle b c\rangle \bar{\lambda}_{b}}{\langle a c\rangle} \lambda_{a}, \quad \ell_{4}=\frac{\langle c a\rangle \bar{\lambda}_{c}+\langle b a\rangle \bar{\lambda}_{b}}{\langle c a\rangle} \lambda_{c} .
\end{aligned}
$$

So that

$$
\begin{aligned}
A_{3}^{(0)}\left(a, \ell_{2}, \ell_{1}\right) A_{3}^{(0)}\left(b, \ell_{3}, \ell_{2}\right) A_{3}^{(0)}\left(c, \ell_{4}, \ell_{3}\right) \\
\quad \times A_{4: 1}^{(1)}\left(\ell_{4}, d, e, \ell_{1}\right) \\
=\frac{2 i}{3} s_{a b} s_{b c} \times \frac{[d e]^{2}}{\langle a b\rangle\langle b c\rangle\langle c a\rangle} \\
=\frac{1}{3} s_{a b} s_{b c} \times A_{5: 3}^{(1)}(d, e ; a, b, c) .
\end{aligned}
$$

Consequently,

$$
P_{5: \lambda}^{(2)} \sim \sum A_{5: 3}^{(1)}(d, e ; a, b, c) \times \mathrm{F}_{a b c ; d e}^{1 m}
$$

where $\mathrm{F}_{a b c ; d e}^{1 m} \equiv \mathrm{F}^{1 m}\left[s_{a b}, s_{b c}, s_{d e}\right]$. We can determine the terms in the summation by expanding $\mathcal{C}^{\prime}$ using $U\left(N_{c}\right)$ identities:

$$
\begin{aligned}
& \mathcal{C}_{(d e ; a b c)}^{\prime}=\sum_{m, n, p, q}((\operatorname{Tr}[a m n]-\operatorname{Tr}[m a n])(\operatorname{Tr}[b p n]-\operatorname{Tr}[p b n])(\operatorname{Tr}[p c q]-\operatorname{Tr}[p q c]))\left(N_{c} \operatorname{Tr}[\text { mqed }]+N_{c} \operatorname{Tr}[\text { meqd }] / 2\right. \\
& +N_{c} \operatorname{Tr}[\text { qemd }] / 2+N_{c} \operatorname{Tr}[\text { qmed }]-3 \operatorname{Tr}[m] \operatorname{Tr}[q d e]-3 \operatorname{Tr}[q] \operatorname{Tr}[m d e]-3 \operatorname{Tr}[d] \operatorname{Tr}[\text { emq }]-3 \operatorname{Tr}[d] \operatorname{Tr}[\text { eqm }] \\
& +3 \operatorname{Tr}[d e] \operatorname{Tr}[m q]+3 \operatorname{Tr}[d m] \operatorname{Tr}[e q]+3 \operatorname{Tr}[d q] \operatorname{Tr}[e m]+\{d \leftrightarrow e\}) \\
& =N_{c}^{2}(\operatorname{Tr}[\text { deabc }]+\operatorname{Tr}[e d a b c]-\operatorname{Tr}[\text { badec }]-\operatorname{Tr}[\text { baedc }])+N_{c}(-2 \operatorname{Tr}[d](\operatorname{Tr}[e a b c]-\operatorname{Tr}[\text { baec }]) \\
& -2 \operatorname{Tr}[e](\operatorname{Tr}[d a b c]-\operatorname{Tr}[b a d c])-\operatorname{Tr}[a](\operatorname{Tr}[d e b c]+\operatorname{Tr}[e d b c]-\operatorname{Tr}[b d e c]-\operatorname{Tr}[b e d c])-\operatorname{Tr}[b](\operatorname{Tr}[d e a c] \\
& +\operatorname{Tr}[e d a c]-\operatorname{Tr}[a e d c]-\operatorname{Tr}[a d e c])-\operatorname{Tr}[c](\operatorname{Tr}[\text { deab }]+\operatorname{Tr}[e d a b]-\operatorname{Tr}[a d e b]-\operatorname{Tr}[a e d b])+8 \operatorname{Tr}[d e](\operatorname{Tr}[a b c] \\
& -\operatorname{Tr}[b a c])+\operatorname{Tr}[d a](\operatorname{Tr}[b e c]-\operatorname{Tr}[e b c])+\operatorname{Tr}[d b](\operatorname{Tr}[a e c]-\operatorname{Tr}[e a c])+\operatorname{Tr}[d c](\operatorname{Tr}[a e b]-\operatorname{Tr}[e a b]) \\
& -\operatorname{Tr}[e a](\operatorname{Tr}[d b c]-\operatorname{Tr}[b d c])-\operatorname{Tr}[e b](\operatorname{Tr}[d a c]-\operatorname{Tr}[a d c])-\operatorname{Tr}[e c](\operatorname{Tr}[d a b]-\operatorname{Tr}[a d b])) \\
& +3(-2 \operatorname{Tr}[d] \operatorname{Tr}[e](\operatorname{Tr}[a b c]-\operatorname{Tr}[b a c])+\operatorname{Tr}[d] \operatorname{Tr}[a](\operatorname{Tr}[e b c]-\operatorname{Tr}[b e c])+\operatorname{Tr}[d] \operatorname{Tr}[b](\operatorname{Tr}[e a c]-\operatorname{Tr}[a e c]) \\
& +\operatorname{Tr}[d] \operatorname{Tr}[c](\operatorname{Tr}[e a b]-\operatorname{Tr}[a e b])+\operatorname{Tr}[e] \operatorname{Tr}[a](\operatorname{Tr}[d b c]-\operatorname{Tr}[b d c])+\operatorname{Tr}[e] \operatorname{Tr}[b](\operatorname{Tr}[d a c]-\operatorname{Tr}[a d c]) \\
& +\operatorname{Tr}[e] \operatorname{Tr}[c](\operatorname{Tr}[d a b]-\operatorname{Tr}[a d b])) \\
& +6(\operatorname{Tr}[\text { deabc }]-\operatorname{Tr}[\text { dcbae }]+\operatorname{Tr}[\text { dcbea }]-\operatorname{Tr}[\text { daebc }]+\operatorname{Tr}[\text { dceba }]-\operatorname{Tr}[\text { dabec }]+\operatorname{Tr}[\text { dcaeb }]-\operatorname{Tr}[\text { dbeac }] \\
& +\operatorname{Tr}[\text { dbaec }]-\operatorname{Tr}[\text { dceab }]+\operatorname{Tr}[\text { dabce }]-\operatorname{Tr}[\text { decba }]+\operatorname{Tr}[\text { daecb }]-\operatorname{Tr}[\text { dbcea }]+\operatorname{Tr}[\text { dbeca }]-\operatorname{Tr}[\text { daceb }]) .
\end{aligned}
$$

This is an expansion of the form

$$
C_{(d e ; a b c)}^{\prime}=\sum_{\lambda} a_{(d e ; a b c)}^{\lambda} C^{\lambda}
$$

where the $C^{\lambda}$ are the different color structures. Consequently the polylogarithmic part of the partial amplitudes is

$P_{5: \lambda}^{(2)}=\sum_{(d e ; a b c)} a_{(d e ; a b c)}^{\lambda} A_{5: 3}(d, e ; a, b, c) \times \mathrm{F}_{a b c ; d e}^{1 m}$.

Specifically we recover the previous results of Refs. [5,13]. Defining $S_{5: 1}=Z_{5}(a, b, c, d, e), S_{5: 2}=Z_{4}(b, c, d, e)$ and $S_{5: 3}=Z_{2}(a, b) \times Z_{3}(c, d, e)$ we have

$$
\begin{aligned}
P_{5: 1}^{(2)}(a, b, c, d, e)= & \sum_{S_{5: 1}}-A_{5: 3}^{(1)}(d, e ; a, b, c) \mathrm{F}_{a b c ; d e}^{1 m}, \\
P_{5: 3}^{(2)}(a, b: c, d, e)= & \frac{4}{3} \sum_{S_{5: 3}}\left(A_{5: 3}^{(1)}(a, b ; c, d, e) \mathrm{F}_{c d e, a b}^{1 m}\right. \\
& +\frac{1}{4} A_{5: 3}^{(1)}(a, c ; b, e, d) \\
& \left.\times\left(\mathrm{F}_{b e d ; a c}^{1 m}+\mathrm{F}_{b d e ; a c}^{1 m}-\mathrm{F}_{d b e ; a c}^{1 m}\right)\right) .
\end{aligned}
$$

We also determine directly the remaining $S U\left(N_{c}\right)$ partial amplitude, 


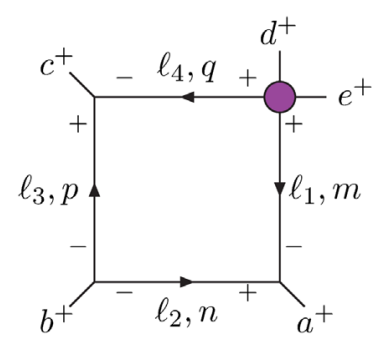

FIG. 3. The labeling and internal helicities of the quadruple cut.

$$
\begin{aligned}
& P_{5: 1 B}^{(2)}(a, b, c, d, e) \\
& \quad=2 \sum_{S_{5: 1}}\left(A_{5: 3}^{(1)}(a, b ; c, d, e) \mathrm{F}_{c d e ; a b}^{1 m}\right. \\
& \left.\quad+A_{5: 3}^{(1)}(a, c ; b, e, d)\left(\mathrm{F}_{b e d ; a c}^{1 m}+\mathrm{F}_{b d e ; a c}^{1 m}-\mathrm{F}_{d b e ; a c}^{1 m}\right)\right) .
\end{aligned}
$$

This expression matches that obtained by using the results of Eq. (5.19) in Eq. (3.6).

The specifically $U\left(N_{c}\right)$ partial amplitudes may also be extracted directly:

$$
\begin{aligned}
P_{5: 2}^{(2)}( & a ; b, c, d, e) \\
= & -\frac{2}{3} \sum_{S_{5: 2}}\left(A_{5: 3}^{(1)}(a, b ; c, d, e) \mathrm{F}_{c d e ; a b}^{1 m}\right. \\
& \left.+\frac{1}{2} A_{5: 3}^{(1)}(b, c ; a, e, d)\left(\mathrm{F}_{a d e ; b c}^{1 m}+\mathrm{F}_{d e a ; b c}^{1 m}-\mathrm{F}_{d a e ; b c}^{1 m}\right)\right)
\end{aligned}
$$

and

$$
\begin{aligned}
& P_{5: 1,1}^{(2)}(a ; b ; c, d, e) \\
& =-\sum_{S_{5: 3}}\left(A_{5: 3}^{(1)}(a, b ; c, d, e) \mathrm{F}_{c d e ; a b}^{1 m}\right. \\
& \left.\quad+A_{5: 3}^{(1)}(a, c ; b, e, d)\left(\mathrm{F}_{b e d ; a c}^{1 m}+\mathrm{F}_{b d e ; a c}^{1 m}-\mathrm{F}_{d b e ; a c}^{1 m}\right)\right) .
\end{aligned}
$$

As a check we have confirmed that these satisfy the decoupling identities (3.4).

\section{RECURSION}

The remaining part of the amplitude is the rational function $R_{n: \lambda}^{(2)}$. In Eq. [8] we described a technique for evaluating this for the leading in color partial amplitude. We review this here and describe the extensions necessary to determine the full-color amplitude.

As $R_{n: \lambda}^{(2)}$ is a rational function we can obtain it recursively given sufficient information about its singularities. BrittoCachazo-Feng-Witten (BCFW) [29] exploited the analytic properties of $n$-point tree amplitudes under a complex shift of their external momenta to compute these amplitudes recursively. Explicitly the BCFW shift acting on two momenta, say $p_{a}$ and $p_{b}$, is

$$
\bar{\lambda}_{a} \rightarrow \bar{\lambda}_{\hat{a}}=\bar{\lambda}_{a}-z \bar{\lambda}_{b}, \quad \lambda_{b} \rightarrow \lambda_{\hat{b}}=\lambda_{b}+z \lambda_{a} .
$$

This introduces a complex parameter, $z$, while preserving overall momentum conservation and keeping all external momenta null. Alternative shifts can also be employed, for example the Risager shift [30] which acts on three momenta, say $p_{a}, p_{b}$ and $p_{c}$, to give

$$
\begin{aligned}
\lambda_{a} & \rightarrow \lambda_{\hat{a}}=\lambda_{a}+z[b c] \lambda_{\eta}, \\
\lambda_{b} & \rightarrow \lambda_{\hat{b}}=\lambda_{b}+z[c a] \lambda_{\eta}, \\
\lambda_{c} & \rightarrow \lambda_{\hat{c}}=\lambda_{c}+z[a b] \lambda_{\eta},
\end{aligned}
$$

where $\lambda_{\eta}$ must satisfy $\langle a \eta\rangle \neq 0$ etc., but is otherwise unconstrained.

After applying either of these shifts, the rational quantity of interest is a complex function parametrized by $z$ i.e., $R(z)$. If $R(z)$ vanishes at large $|z|$, then Cauchy's theorem applied to $R(z) / z$ over a contour at infinity implies

$$
R=R(0)=-\left.\sum_{z_{j} \neq 0} \operatorname{Res}\left[\frac{R(z)}{z}\right]\right|_{z_{j}} .
$$

If the function only contains simple poles, $\left.\operatorname{Res}[R(z) / z]\right|_{z_{j}}=$ $\left.\operatorname{Res}[R(z)]\right|_{z_{j}} / z_{j}$ and we can use factorization theorems to determine the residues. Higher order poles do not present a problem mathematically, for example, given a function with a double pole at $z=z_{j}$ and its Laurent expansion,

$$
R(z)=\frac{c_{-2}}{\left(z-z_{j}\right)^{2}}+\frac{c_{-1}}{\left(z-z_{j}\right)}+\mathcal{O}\left(\left(z-z_{j}\right)^{0}\right),
$$

the residue is simply

$$
\left.\operatorname{Res}\left[\frac{R(z)}{z}\right]\right|_{z_{j}}=-\frac{c_{-2}}{z_{j}^{2}}+\frac{c_{-1}}{z_{j}} .
$$

To determine this we need to know both the leading and subleading poles. As discussed above, loop amplitudes can contain double poles and, at this point, there are no general theorems determining the subleading pole.

Both the BCFW and Risager shifts break cyclic symmetry of the amplitude by acting on specific legs and the Risager shift further introduces the arbitrary spinor $\eta$. While it is hard to determine a priori the large $z$ behavior of an unknown amplitude, recovering cyclic symmetry (and $\eta$ independence) are powerful checks. For the two-loop allplus amplitude this symmetry recovery does not occur for the BCFW shift (the one-loop all-plus amplitudes have the same feature). However, symmetry is recovered if we employ the Risager shift (6.2).

The Risager shift excites poles corresponding to tree: two-loop and one-loop:one-loop factorizations. The former involve only single poles and their contributions are readily 
obtained from the rational parts of the four-point two-loop amplitude [2]:

$$
\begin{aligned}
R_{4: 1}^{(2)}\left(K^{+}, c^{+}, d^{+}, e^{+}\right) & =\frac{1}{3} A_{4: 1}^{(1)}\left(K^{+}, c^{+}, d^{+}, e^{+}\right)\left(\frac{s_{c e}^{2}}{s_{c d} s_{d e}}+8\right), \\
R_{4: 3}^{(2)}\left(K^{+}, c^{+} ; d^{+}, e^{+}\right)= & \frac{1}{9} A_{4: 3}^{(1)}\left(K^{+}, c^{+} ; d^{+}, e^{+}\right) \\
& \times\left(\frac{s_{c d}^{2}}{s_{c e} s_{d e}}+\frac{s_{c e}^{2}}{s_{c d} s_{d e}}+\frac{s_{d e}^{2}}{s_{c d} s_{c e}}+24\right) .
\end{aligned}
$$

The one-loop:one-loop factorizations involve double poles and we need to determine the subleading pieces. By considering a diagram of the form Fig. 4 using an axial gauge formalism, we can determine the full pole structure of the rational piece, including the nonfactorizing simple poles. We have used this approach previously to compute one-loop [31-33] and two-loop amplitudes [6-8]; we labeled this process augmented recursion. In axial gauge formalism helicity labels can be assigned to internal offshell legs and vertices expressed in terms of nullified momenta [34,35],

$$
K^{b}=K-\frac{K^{2}}{2 K \cdot q} q
$$

where $q$ is a reference momentum. The two off-shell legs are

$$
\alpha=\alpha(\ell)=\ell+a \quad \text { and } \quad \beta=\beta(l)=b-\ell,
$$

where we also define the sum of these legs, $P_{\alpha \beta}=$ $\alpha+\beta=a+b=P_{a b}$, which is independent of the loop momentum, $\ell$.

The principal helicity assignment in Fig. 4, gives

$$
\int d \Lambda^{c}\left(\alpha^{+}, a^{+}, b^{+}, \beta^{-}\right) \tau_{n}^{(1), c}\left(\alpha^{-}, \beta^{+}, c^{+}, \ldots, n^{+}\right),
$$

where

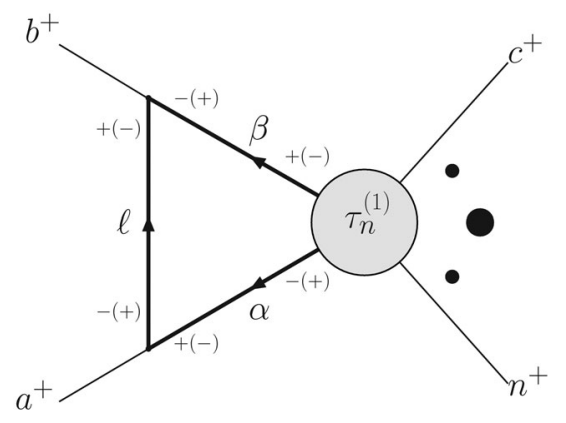

FIG. 4. Diagram containing the leading and sub-leading poles as $s_{a b} \rightarrow 0$. The axial gauge construction permits the off-shell continuation of the internal legs.

$$
\begin{aligned}
& \int d \Lambda^{c}\left(\alpha^{+}, a^{+}, b^{+}, \beta^{-}\right) \\
& \equiv \frac{i}{c_{\Gamma}(2 \pi)^{D}} \int \frac{d^{D} \ell}{\ell^{2} \alpha^{2} \beta^{2}} \mathcal{V}_{3}(\alpha, a, \ell) \mathcal{V}_{3}(\ell, b, \beta),
\end{aligned}
$$

the vertices are in axial gauge and $\tau_{n}^{(1), c}$ is a doubly off-shell current.

As we are only interested in the residue on the $s_{a b} \rightarrow 0$ pole, we do not need the exact current. It is sufficient that the approximate current satisfies two conditions [6,32]:

(C1) The current contains the leading singularity as $s_{\alpha \beta} \rightarrow 0$ with $\alpha^{2}, \beta^{2} \neq 0$,

(C2) The current is the one-loop, single-minus amplitude in the on-shell limit $\alpha^{2}, \beta^{2} \rightarrow 0, s_{\alpha \beta} \neq 0$.

This process is detailed in Ref. [8].

We now apply the method to the full color amplitude. The $U\left(N_{c}\right)$ color decomposition of $d \Lambda^{c}$ contains a common kinematic factor so we have the color decompositions

$\tau_{n}^{(1), c}=\sum_{\lambda} C_{\lambda} \tau_{n: \lambda}^{(1)} \quad$ and $\quad \int d \Lambda^{c}=C_{\Lambda} \int d \Lambda_{0}$

where

$$
\begin{aligned}
& \int d \Lambda_{0}\left(\alpha^{+}, a^{+}, b^{+}, \beta^{-}\right) \\
& =\frac{i}{c_{\Gamma}(2 \pi)^{D}} \int \frac{d^{D} \ell}{\ell^{2} \alpha^{2} \beta^{2}} \frac{[a|\ell| q\rangle[b|\ell| q\rangle}{\langle a q\rangle\langle b q\rangle} \frac{\langle\beta q\rangle^{2}}{\langle\alpha q\rangle^{2}} .
\end{aligned}
$$

Hence the full color contribution is

$\sum_{\lambda} C_{\Lambda} C_{\lambda} \int d \Lambda_{0}\left(\alpha^{+}, a^{+}, b^{+}, \beta^{-}\right) \tau_{n: \lambda}^{(1)}\left(\alpha^{-}, \beta^{+}, c^{+}, \ldots n^{+}\right)$.

The various $\tau_{n: \lambda}^{(1)}$ can be expressed as sums of the leading amplitudes $\tau_{n: 1}^{(1)}$ via a series of $U(1)$ decoupling identities.

We now focus on the five-point case, where there are two distinct forms of the leading current,

$\tau_{5: 1}^{(1)}\left(\alpha^{-}, \beta^{+}, c^{+}, d^{+}, e^{+}\right) \quad$ and $\quad \tau_{5: 1}^{(1)}\left(\alpha^{-}, c^{+}, \beta^{+}, d^{+}, e^{+}\right)$,

which we call the "adjacent" and "non-adjacent" leading currents, respectively.

$\tau_{5: 1}^{(1)}\left(\alpha^{-}, \beta^{+}, c^{+}, d^{+}, e^{+}\right)$has been calculated previously for a specific choice of the axial gauge spinor $\lambda_{q}=\lambda_{d}$ [6]. Since we require currents for which all the legs have been permuted it is necessary to derive this current for arbitrary $\lambda_{q}$. The nonadjacent case has not previously been considered. The derivation of the adjacent current is given in Appendix B. This current is given by 


$$
\begin{aligned}
\tau_{5: 1}^{(1)}\left(\alpha^{-}, \beta^{+}, c^{+}, d^{+}, e^{+}\right)= & \mathcal{F}_{d p}^{c d e}\left[1+s_{\alpha \beta}\left(\frac{[q e]}{[c e]\left[q\left|P_{\alpha \beta}\right| c\right\rangle}+\frac{[c|q| d\rangle}{\left[q\left|P_{\alpha \beta}\right| q\right\rangle[c|e| d\rangle}+\frac{[e|q| d\rangle}{\left[q\left|P_{\alpha \beta}\right| q\right\rangle[e|c| d\rangle}\right)\right] \\
& +\frac{i}{3\langle c d\rangle^{2}} \frac{\langle\alpha q\rangle^{2}}{\langle\beta q\rangle^{2}}\left[\frac{\langle\alpha c\rangle[c|\beta| d\rangle}{\langle d e\rangle\langle e \alpha\rangle}+\frac{\langle c e\rangle[d e]}{\langle d e\rangle^{2}}\left(\frac{\left[q\left|P_{\alpha \beta}\right| d\right\rangle^{3}}{\left[q q P_{\alpha \beta}|q\rangle^{3}\right.} \frac{\langle q c\rangle\langle q \alpha\rangle[q|\alpha| q\rangle}{\langle\alpha c\rangle\left[q\left|P_{\alpha \beta}\right| c\right\rangle}-3 \frac{\langle q d\rangle\left[q\left|P_{\alpha \beta}\right| d\right\rangle^{2}[q|\beta| q\rangle}{\left[q\left|P_{\alpha \beta}\right| q\right\rangle^{2}\left[q\left|P_{\alpha \beta}\right| c\right\rangle}\right)\right] \\
& +\mathcal{F}_{s b}^{c d e}+\frac{i}{3\langle c d\rangle^{2}}\left(-\frac{[\beta e]^{2}[q e]}{[e \alpha][\alpha q]}+[e|q| \alpha\rangle \frac{\left([e \beta][\beta q]\left[q\left|P_{\alpha \beta}\right| q\right\rangle-[\beta q]^{2}\left[e\left|P_{\alpha \beta}\right| q\right\rangle\right)}{[\alpha q]\left[q\left|P_{\alpha \beta}\right| q\right\rangle^{2}}\right) \\
& +\mathcal{O}(\langle\alpha \beta\rangle)+\mathcal{O}\left(\alpha^{2}\right)+\mathcal{O}\left(\beta^{2}\right)
\end{aligned}
$$

where

$$
\mathcal{F}_{d p}^{c d e}=\frac{i}{3} \frac{\langle\alpha q\rangle^{2}}{\langle\beta q\rangle^{2}} \frac{\langle q|\alpha \beta| q\rangle}{s_{\alpha \beta}} \frac{\langle e c\rangle[c e]^{3}}{\langle c d\rangle\langle d e\rangle\left[e\left|P_{\alpha \beta}\right| q\right\rangle\left[c\left|P_{\alpha \beta}\right| q\right\rangle}
$$

and

$$
\mathcal{F}_{s b}^{c d e}=-\frac{i}{3} \frac{\left[e\left|P_{\alpha \beta}\right| \alpha\right\rangle[\beta q]^{2}}{[\alpha q]\left[q\left|P_{\alpha \beta}\right| q\right\rangle} \frac{1}{s_{\alpha \beta}} \frac{\left[e\left|P_{\alpha \beta}\right| q\right\rangle}{\langle c d\rangle^{2}}
$$

Setting $\lambda_{q}=\lambda_{d}$ in Eq. (6.14) reproduces the current presented in Ref. [6].

The nonadjacent leading current is

$$
\begin{aligned}
& \tau_{5: 1}^{(1)}\left(\alpha^{-}, c^{+}, \beta^{+}, d^{+}, e^{+}\right) \\
& \quad=\frac{i}{3} \frac{\langle\alpha q\rangle^{2}}{\langle\beta q\rangle^{2}}\left(\frac{\langle\alpha e\rangle[e c]}{\langle c \alpha\rangle\langle d e\rangle^{2}}-\frac{[e c]^{3}}{[e|\alpha| d\rangle[c|\alpha| d\rangle}\right)+\mathcal{O}(\langle\alpha \beta\rangle) .
\end{aligned}
$$

These currents must be integrated before extracting the rational pole. The nonadjacent case integrates to the simple form,

$$
\int \frac{d^{D} \ell}{\ell^{2} \alpha^{2} \beta^{2}} \frac{i}{3} \frac{[a|\ell| q\rangle[b|\ell| q\rangle}{\langle a q\rangle\langle b q\rangle} \frac{\langle a e\rangle[e c]}{\langle c a\rangle\langle d e\rangle^{2}}=\frac{i}{6} \frac{[e c]\langle a e\rangle[a b]}{\langle d e\rangle^{2}\langle c a\rangle\langle a b\rangle}
$$

where the second term in Eq. (6.17) has been dropped since it is a quadratic pentagon and does not contain any rational terms. The integrated adjacent case is a generalization of the previous result [6]. Summing over all the channels excited by the Risager shift we recover the full two-loop color decomposition. We present compact forms of the $S U\left(N_{c}\right)$ rational pieces below, including the first compact form for the rational piece of the maximally nonplanar amplitude obtained via a direct computation. We find complete agreement with previous calculations [13] and $R_{5: 1 B}^{(2)}$ satisfies the constraint (3.6).

$$
\begin{aligned}
R_{5: 1}^{(2)} & \left(a^{+}, b^{+}, c^{+}, d^{+}, e^{+}\right) \\
= & \frac{i}{9} \frac{1}{\langle a b\rangle\langle b c\rangle\langle c d\rangle\langle d e\rangle\langle e a\rangle} \\
& \times \sum_{S_{5: 1}}\left(\frac{\operatorname{tr}_{+}^{2}[d e a b]}{s_{d e} s_{a b}}+5 s_{a b} s_{b c}+s_{a b} s_{c d}\right),
\end{aligned}
$$

$$
\begin{aligned}
R_{5: 3}^{(2)}( & \left.a^{+}, b^{+} ; c^{+}, d^{+}, e^{+}\right) \\
= & -\frac{2 i}{3} \frac{1}{\langle a b\rangle\langle b a\rangle\langle c d\rangle\langle d e\rangle\langle e c\rangle} \\
& \times \sum_{S_{5: 3}}\left(\frac{\operatorname{tr}_{-}[a c d e] \operatorname{tr}_{-}[e c b a]}{s_{a e} s_{c d}}+\frac{3}{2} s_{a b}^{2}\right)
\end{aligned}
$$

and

$$
\begin{aligned}
R_{5: 1 B}^{(2)} & \left(a^{+}, b^{+}, c^{+}, d^{+}, e^{+}\right) \\
= & 2 i \epsilon(a, b, c, d)\left(\mathrm{C}_{\mathrm{PT}}(a, b, e, c, d)\right. \\
& +\mathrm{C}_{\mathrm{PT}}(a, d, b, c, e)+\mathrm{C}_{\mathrm{PT}}(b, c, a, d, e) \\
& \left.+\mathrm{C}_{\mathrm{PT}}(a, b, d, e, c)+\mathrm{C}_{\mathrm{PT}}(a, c, d, b, e)\right)
\end{aligned}
$$

where

$$
\mathrm{C}_{\mathrm{PT}}(a, b, c, d, e)=\frac{1}{\langle a b\rangle\langle b c\rangle\langle c d\rangle\langle d e\rangle\langle e a\rangle} .
$$

These expressions are valid for both $U\left(N_{c}\right)$ and $S U\left(N_{c}\right)$ gauge groups and are remarkably compact.

We note that there are double poles at leading and subleading in color, but not at sub-sub-leading. As $R_{4: 1 B}^{(2)}$ vanishes [2] the poles in $R_{5: 1 B}^{(2)}$ do not correspond to tree: two-loop factorizations, instead they arise from contributions of the type shown in Fig. 4, where the corresponding current has no pole in $s_{a b}$.

\section{CONCLUSIONS}

Computing perturbative gauge theory amplitudes to high orders is an important but difficult task. In this article, we have demonstrated how the full color all-plus five-point amplitude may be computed in simple forms. We have computed all the color components directly and only used 
color relations between them as checks. In passing, we have given simple all- $n$ expressions for the one-loop subleading in color amplitudes and presented the $n$-point IR divergences in a color basis approach. Our methodology obtains these results without the need to determine two-loop nonplanar integrals.

\section{ACKNOWLEDGMENTS}

D. C. D. was supported by STFC Grant No. ST/L000369/1. J. M. W. S. was supported by STFC Grant No. ST/S505778/1. J. H. G. was supported by the College of Science Doctoral Training Centre at Swansea University.

\section{APPENDIX A: INFRARED DIVERGENCES}

The singular behavior of two-loop gluon scattering amplitudes is known from a general analysis [23]. The leading IR singularity for the $n$-point two-loop amplitude is [36]

$$
-\frac{s_{a b}^{-\epsilon}}{\epsilon^{2}} f^{a i j} f^{b i k} \times \mathcal{A}_{n}^{(1)}(j, k, \ldots, n)
$$

where $\mathcal{A}_{n}^{(1)}$ is the full-color one-loop amplitude. We wish to disentangle this simple equation into the color-ordered partial amplitudes. It will be convenient to use a more list based notation for the partial amplitudes where we use

$A_{n}^{(l)}(S)=A_{n}^{(l)}\left(\left\{a_{1}, a_{2}, \ldots a_{n}\right\}\right) \equiv A_{n: 1}^{(l)}\left(a_{1}, a_{2}, \ldots a_{n}\right)$,

$A_{n}^{(l)}\left(S_{1} ; S_{2}\right)$ for $A_{n: r}^{(l)}$ and $A_{n}^{(l)}\left(S_{1} ; S_{2} ; S_{3}\right)$ for $A_{n: s, t}^{(l)}$.

First we define

$$
I_{i, j} \equiv-\frac{\left(s_{i j}\right)^{-\epsilon}}{\epsilon^{2}}
$$

and we have for a list $S=\left\{a_{1}, a_{2}, a_{3}, \ldots, a_{r}\right\}$,

$$
I_{r}[S]=\sum_{i=1}^{r} I_{a_{i}, a_{i+1}}
$$

where the term $I_{a_{r}, a_{r+1}} \equiv I_{a_{r}, a_{1}}$ is included in the sum. We also define $I_{j}\left[S_{1}, S_{2}\right]$ and $I_{k}\left[S_{1}, S_{2}\right]$,

$$
\begin{aligned}
I_{j}\left[S_{1}, S_{2}\right] & =I_{j}\left[\left\{a_{1}, a_{2} \ldots a_{r}\right\},\left\{b_{1}, b_{2}, \ldots b_{s}\right\}\right] \\
& \equiv\left(I_{a_{1}, a_{r}}+I_{b_{1}, b_{s}}-I_{a_{1}, b_{1}}-I_{a_{r}, b_{s}}\right), \\
I_{k}\left[S_{1}, S_{2}\right] & =I_{k}\left[\left\{a_{1}, a_{2} \ldots a_{r}\right\},\left\{b_{1}, b_{2}, \ldots b_{s}\right\}\right] \\
& \equiv\left(I_{a_{1}, b_{s}}+I_{b_{1}, a_{r}}-I_{a_{1}, b_{1}}-I_{a_{r}, b_{s}}\right)
\end{aligned}
$$

giving

$I_{r}\left[S_{1} \oplus S_{2}\right]=I_{r}\left[S_{1}\right]+I_{r}\left[S_{2}\right]+I_{k}\left[S_{1}, S_{2}\right]-I_{j}\left[S_{1}, S_{2}\right]$

where $\left\{a_{1} \ldots a_{r}\right\} \oplus\left\{b_{1} \ldots b_{s}\right\}=\left\{a_{1} \ldots a_{r}, b_{1} \ldots b_{s}\right\}$. In this language the leading and subeading IR singularities at one-loop are

$$
\begin{aligned}
A_{n}^{(1)}(S) & =A_{n}^{(0)}(S) \times I_{r}[S], \\
A_{n}^{(1)}\left(S_{1} ; S_{2}\right) & =\sum_{S_{1}^{\prime} \in C\left(S_{1}\right)} \sum_{S_{2}^{\prime} \in C\left(S_{2}\right)} A_{n}^{(0)}\left(S_{1}^{\prime} \oplus S_{2}^{\prime}\right) \times I_{j}\left[S_{1}^{\prime}, S_{2}^{\prime}\right] .
\end{aligned}
$$

The set $C(S)$ is the set of cyclic permutations of $S$.

At two-loops, we have

$$
\begin{aligned}
A_{n}^{(2)}(S)= & A_{n}^{(1)}(S) \times I_{r}[S], \\
A_{n}^{(2)}\left(S_{1} ; S_{2}\right)= & A_{n}^{(1)}\left(S_{1} ; S_{2}\right) \times\left(I_{r}\left[S_{1}\right]+I_{r}\left[S_{2}\right]\right)+\sum_{S_{1}^{\prime} \in C\left(S_{1}\right)} \sum_{S_{2}^{\prime} \in C\left(S_{2}\right)} A_{n}^{(1)}\left(S_{1}^{\prime} \oplus S_{2}^{\prime}\right) \times I_{j}\left[S_{1}^{\prime}, S_{2}^{\prime}\right], \\
A_{n}^{(2)}\left(S_{1} ; S_{2} ; S_{3}\right)= & \sum_{S_{2}^{\prime} \in C\left(S_{2}\right)} \sum_{S_{3}^{\prime} \in C\left(S_{3}\right)} A_{n}^{(1)}\left(S_{1} ; S_{2}^{\prime} \oplus S_{3}^{\prime}\right) \times I_{j}\left[S_{2}^{\prime}, S_{3}^{\prime}\right]+\sum_{S_{1}^{\prime} \in C\left(S_{1}\right)} \sum_{S_{3}^{\prime} \in C\left(S_{3}\right)} A_{n}^{(1)}\left(S_{2} ; S_{1}^{\prime} \oplus S_{3}^{\prime}\right) \times I_{j}\left[S_{1}^{\prime}, S_{3}^{\prime}\right] \\
& +\sum_{S_{1}^{\prime} \in C\left(S_{1}\right)} \sum_{S_{2}^{\prime} \in C\left(S_{2}\right)} A_{n}^{(1)}\left(S_{3} ; S_{1}^{\prime} \oplus S_{2}^{\prime}\right) \times I_{j}\left[S_{1}^{\prime}, S_{2}^{\prime}\right], \\
A_{n, B}^{(2)}(S)= & \sum_{U(S)} A_{n}^{(1)}\left(S_{1}^{\prime} ; S_{2}^{\prime}\right) \times I_{k}\left[S_{1}^{\prime}, S_{2}^{\prime}\right],
\end{aligned}
$$

where $U(S)$ is the set of all distinct pairs of lists satisfying $S_{1}^{\prime} \oplus S_{2}^{\prime} \in C(S)$ where the size of $S_{i}^{\prime}$ is greater than one. For example

$$
U(\{1,2,3,4,5\})=\{(\{1,2\},\{3,4,5\}),(\{2,3\},\{4,5,1\}),(\{3,4\},\{5,1,2\}),(\{4,5\},\{1,2,3\}),(\{5,1\},\{2,3,4\})\} .
$$




\section{APPENDIX B: OBTAINING THE ADJACENT CURRENT}

We build the rational part of the full color five-point amplitude recursively, using augmented recursion to determine the subleading poles arising in the one-loop:one-loop factorizations. For this we need an approximation to the doubly massive current $\tau_{5}^{(1)}(\alpha, \beta, c, d, e)$ shown in Fig. 4. As we are only interested in the residue on the $s_{a b} \rightarrow 0$ pole, we do not need the exact current, just one that satisfies the conditions

(C1) The current contains the leading singularity as $s_{\alpha \beta} \rightarrow 0$ with $\alpha^{2}, \beta^{2} \neq 0$,

(C2) The current is the one-loop, single-minus amplitude in the on-shell limit $\alpha^{2}, \beta^{2} \rightarrow 0, s_{\alpha \beta} \neq 0$.

Condition (C2) requires the current $\tau_{5: \lambda}^{(1)}$ to reproduce the full partial amplitude $A_{5: \lambda}^{(1)}$ in the $\alpha^{2} \rightarrow 0, \beta^{2} \rightarrow 0$ limit and so the current should have the same color decomposition as the one-loop amplitude (2.3). We can use (2.6) to relate any of the subleading currents to sums of the leading in color currents $\tau_{5: 1}^{(1)}$. The cyclic and flip symmetries inherited from $A_{5: 1}^{(1)}$ mean that any of the $\tau_{5: \lambda}^{(1)}$ can be related to $\tau_{5: 1}^{(1)}(\alpha, \beta, c, d, e)$ and $\tau_{5: 1}^{(1)}(\alpha, c, \beta, d, e)$ up to permutations of the legs $\{c, d, e\}$.

To build the current we start with the one-loop, fivepoint, single-minus partial amplitude

$A_{5: 1}^{(1)}\left(\alpha^{-}, \beta^{+}, c^{+}, d^{+}, e^{+}\right)=\sum_{j=i, i i, i i i} A_{5: 1 j}^{(1)}\left(\alpha^{-}, \beta^{+}, c^{+}, d^{+}, e^{+}\right)$

where

$$
\begin{aligned}
& A_{5: 1 i}^{(1)}\left(\alpha^{-}, \beta^{+}, c^{+}, d^{+}, e^{+}\right)=\frac{i}{3} \frac{1}{\langle c d\rangle^{2}} \frac{\langle c e\rangle\langle\alpha d\rangle^{3}[d e]}{\langle\alpha \beta\rangle\langle d e\rangle^{2}\langle\beta c\rangle}, \\
& A_{5: 1 i i}^{(1)}\left(\alpha^{-}, \beta^{+}, c^{+}, d^{+}, e^{+}\right)=-\frac{i}{3} \frac{1}{\langle c d\rangle^{2}} \frac{[\beta e]^{3}}{[\alpha \beta][e \alpha]}
\end{aligned}
$$

and

$$
A_{5: 1 i i i}^{(1)}\left(\alpha^{-}, \beta^{+}, c^{+}, d^{+}, e^{+}\right)=\frac{i}{3} \frac{1}{\langle c d\rangle^{2}} \frac{\langle\alpha c\rangle^{3}\langle\beta d\rangle[\beta c]}{\langle d e\rangle\langle\alpha e\rangle\langle\beta c\rangle^{2}} .
$$

Condition (C1) requires our approximation to the current to reproduce the correct leading singularities as $s_{\alpha \beta} \rightarrow 0$, the sources of these are depicted in Fig. 5 [6]. We determine these within the axial gauge formalism. The two channels give

$$
\begin{aligned}
\mathcal{F}_{d p}^{c d e} & \equiv \frac{[\beta k]\langle\alpha q\rangle^{2}}{\langle\beta q\rangle\langle k q\rangle} \frac{1}{s_{\alpha \beta}} A_{4: 1}^{(1)}\left(k^{-}, c^{+}, d^{+}, e^{+}\right) \\
& =\frac{i}{3} \frac{\langle\alpha q\rangle^{2}}{\langle\beta q\rangle^{2}} \frac{\langle q|\alpha \beta| q\rangle}{s_{\alpha \beta}} \frac{\langle e c\rangle[c e]^{3}}{\langle c d\rangle\langle d e\rangle\left[e\left|P_{\alpha \beta}\right| q\right\rangle\left[c\left|P_{\alpha \beta}\right| q\right\rangle}
\end{aligned}
$$

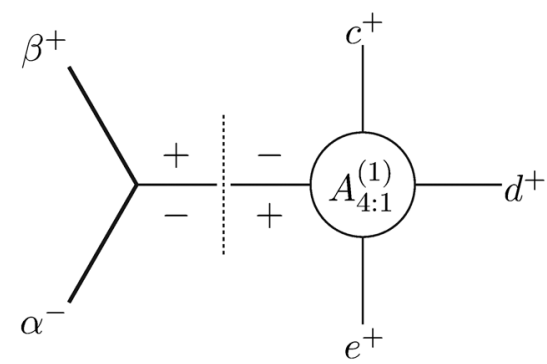

FIG. 5. Factorizations of the current on the $s_{\alpha \beta} \rightarrow 0$ pole.

and

$$
\begin{aligned}
\mathcal{F}_{s b}^{c d e} & \equiv \frac{\langle\alpha k\rangle[\beta q]^{2}}{[\alpha q][k q]} \frac{1}{s_{\alpha \beta}} A_{4: 1}^{(1)}\left(k^{+}, c^{+}, d^{+}, e^{+}\right) \\
& =-\frac{i}{3} \frac{\langle\alpha k\rangle[\beta q]^{2}}{[\alpha q][k q]} \frac{1}{s_{\alpha \beta}} \frac{[e k]^{2}}{\langle c d\rangle^{2}}
\end{aligned}
$$

where $k=\alpha+\beta=-c-d-e$ which is null on the pole.

Using the identity

$$
\begin{aligned}
& \frac{1}{\langle\alpha \beta\rangle\langle\beta c\rangle} \\
& =\frac{1}{\langle\alpha q\rangle\langle\beta q\rangle^{2}}\left(\frac{\langle q|\alpha \beta| q\rangle\left[q\left|P_{\alpha \beta}\right| q\right\rangle}{s_{\alpha \beta}\left[q\left|P_{\alpha \beta}\right| c\right\rangle}+\frac{\langle q \beta\rangle\langle q c\rangle[q|\alpha| q\rangle}{\langle\beta c\rangle\left[q\left|P_{\alpha \beta}\right| c\right\rangle}\right)
\end{aligned}
$$

and the expansion

$\frac{\left[\beta\left|P_{\alpha \beta}^{b}\right| d\right\rangle}{\left[\beta\left|P_{\alpha \beta}\right| q\right\rangle}=\frac{\left[q\left|P_{\alpha \beta}\right| d\right\rangle}{\left[q\left|P_{\alpha \beta}\right| q\right\rangle}+s_{\alpha \beta} \frac{\langle q d\rangle[\beta q]}{\left[\beta\left|P_{\alpha \beta}\right| q\right\rangle\left[q\left|P_{\alpha \beta}\right| q\right\rangle}+\mathcal{O}\left(s_{\alpha \beta}^{2}\right)$

we find

$$
\begin{aligned}
A_{5: 1 i}^{(1)}= & \mathcal{F}_{d p}^{c d e}\left[1+s_{\alpha \beta}\left(\frac{[q e]}{[c e]\left[q\left|P_{\alpha \beta}\right| c\right\rangle}+\frac{[c|q| d\rangle}{\left[q\left|P_{\alpha \beta}\right| q\right\rangle[c|e| d\rangle}\right.\right. \\
& \left.\left.+\frac{[e|q| d\rangle}{\left[q\left|P_{\alpha \beta}\right| q\right\rangle[e|c| d\rangle}\right)+\mathcal{O}\left(s_{\alpha \beta}^{2}\right)\right] .
\end{aligned}
$$

We see that $A_{5: 1 i}^{(1)}$ generates the correct singularity as $\langle\alpha \beta\rangle \rightarrow 0$. This terms generates the double pole when integrated and the form in (B5) explicitly exposes the subleading contribution.

The $\mathcal{F}_{s b}^{c d e}$ factorization arises when $[\alpha \beta] \rightarrow 0$. This we obtain from $A_{5: 1 i i}^{(1)}$. Using

$$
k^{b}=k-\frac{k^{2}}{2 k \cdot q} q=\alpha^{b}+\beta^{b}+\delta q,
$$

where 


$$
\delta=\frac{\alpha^{2}}{2 \alpha \cdot q}+\frac{\beta^{2}}{2 \beta \cdot q}-\frac{s_{\alpha \beta}}{2 k \cdot q}
$$

we have

$$
\begin{aligned}
\mathcal{F}_{s b}^{c d e}= & \frac{i}{3} \frac{1}{s_{\alpha \beta}}\left[\frac{[e \beta]^{2}[\beta q]\langle\beta \alpha\rangle}{[\alpha q]\langle c d\rangle^{2}}\right. \\
& \left.+\delta[e|q| \alpha\rangle \frac{\left([e \beta][\beta q][k q]+[\beta q]^{2}[e k]\right)}{[\alpha q][k q]\langle c d\rangle^{2}}\right] .
\end{aligned}
$$

Now $A_{5: 1 i i}^{(1)}$ can be rewritten as

$$
A_{5: 1 i i}^{(1)}=-\frac{i}{3} \frac{1}{\langle c d\rangle^{2}} \frac{[\beta e]^{2}[q e]}{[e \alpha][\alpha q]}-\frac{i}{3} \frac{1}{\langle c d\rangle^{2}} \frac{[\beta e]^{2}[q \beta]}{[\alpha \beta][\alpha q]}
$$

and noting that

$$
\begin{aligned}
\frac{\langle\beta \alpha\rangle}{s_{\alpha \beta}}-\frac{1}{[\alpha \beta]} & =\frac{\langle\beta \alpha\rangle[\alpha \beta]-s_{\alpha \beta}}{s_{\alpha \beta}[\alpha \beta]}=\frac{\left(\alpha^{b}+\beta^{b}\right)^{2}-s_{\alpha \beta}}{s_{\alpha \beta}[\alpha \beta]} \\
& =-\left(\frac{\alpha^{2}}{2 \alpha \cdot q}+\frac{\beta^{2}}{2 \beta \cdot q}\right) \frac{2 k \cdot q}{s_{\alpha \beta}[\alpha \beta]},
\end{aligned}
$$

we see that $A_{5: 1 i i}^{(1)}$ has the form $\mathcal{F}_{s b}^{c d e}+\Delta_{\alpha^{2}}+\Delta_{\beta^{2}}+\Delta_{s_{\alpha \beta}}$ as $[\alpha \beta] \rightarrow 0$, where $\Delta_{\alpha^{2}}$ is proportional to $\alpha^{2} / s_{\alpha \beta}$ etc.. As $\Delta_{s_{\alpha \beta}}$ does not contribute on the pole, we do not have to replicate it in the current and therefore include a contribution to the current of the form $A_{5: 1 i i}^{(1)}-\Delta_{\alpha^{2}}-\Delta_{\beta^{2}}$ to satisfy condition (C1). This does not compromise condition (C2). Upon integration the $\alpha^{2}$ and $\beta^{2}$ factors in $\Delta_{\alpha^{2}}$ and $\Delta_{\beta^{2}}$ generate $s_{a b}$ factors which cancel the pole. We therefore do not require these forms explicitly. For the purposes of integration it is convenient to express the term with the $[\alpha \beta]$ pole in terms of $\mathcal{F}_{s b}^{c d e}$. To maintain condition $(\mathrm{C} 2)$ we must retain $\Delta_{s_{\alpha \beta}}$.

The remaining piece of the one-loop amplitude, $A_{5: 1 i i i}^{(1)}$, contains no poles as $\langle\alpha \beta\rangle \rightarrow 0$ or $[\alpha \beta] \rightarrow 0$ and we can simplify it using

$$
\frac{\langle X \alpha\rangle}{\langle Y \alpha\rangle}=\frac{\langle X \alpha\rangle}{\langle Y \alpha\rangle} \frac{\langle Y a\rangle}{\langle Y a\rangle}=\frac{\langle X a\rangle}{\langle Y a\rangle}+\mathcal{O}(\langle\alpha a\rangle)
$$

as terms of $\mathcal{O}(\langle\alpha a\rangle)$ do not ultimately contribute to the residue.

The adjacent leading current is then

$$
\begin{aligned}
\tau_{5: 1}^{(1)}\left(\alpha^{-}, \beta^{+}, c^{+}, d^{+}, e^{+}\right)= & \mathcal{F}_{d p}^{c d e}\left[1+s_{\alpha \beta}\left(\frac{[q e]}{[c e]\left[q\left|P_{\alpha \beta}\right| c\right\rangle}+\frac{[c|q| d\rangle}{\left[q\left|P_{\alpha \beta}\right| q\right\rangle[c|e| d\rangle}+\frac{[e|q| d\rangle}{\left[q\left|P_{\alpha \beta}\right| q\right\rangle[e|c| d\rangle}\right)\right] \\
& +\frac{i}{3\langle c d\rangle^{2}} \frac{\langle\alpha q\rangle^{2}}{\langle\beta q\rangle^{2}}\left[\frac{\langle a c\rangle[c|\beta| d\rangle}{\langle d e\rangle\langle e a\rangle}+\frac{\langle c e\rangle[d e]}{\langle d e\rangle^{2}}\left(\frac{\left[q\left|P_{\alpha \beta}\right| d\right\rangle^{3}\langle q c\rangle\langle q a\rangle[q|\alpha| q\rangle}{\left[q\left|P_{\alpha \beta}\right| q\right\rangle^{3}} \frac{\langle q d\rangle\left[q\left|P_{\alpha \beta}\right| d\right\rangle^{2}[q|\beta| q\rangle}{\left.\langle a c\rangle\left|P_{\alpha \beta}\right| c\right\rangle}\right)\right] \\
& +\mathcal{F}_{s b}^{c d e}+\frac{i}{3\langle c d\rangle^{2}}\left(-\frac{[\beta e]^{2}[q e]}{[e \alpha][\alpha q]}+[e|q| \alpha\rangle \frac{\left([e \beta][\beta q][k q]+[\beta q]^{2}[e k]\right)}{[\alpha q][k q] 2 k . q}\right)+\mathcal{O}(\langle\alpha \beta\rangle)+\mathcal{O}\left(\alpha^{2}\right)+\mathcal{O}\left(\beta^{2}\right) .
\end{aligned}
$$

[1] E. W. N. Glover, C. Oleari, and M. E. Tejeda-Yeomans, Two loop QCD corrections to gluon-gluon scattering, Nucl. Phys. B605, 467 (2001).

[2] Z. Bern, A. De Freitas, and L. J. Dixon, Two loop helicity amplitudes for gluon-gluon scattering in QCD and supersymmetric Yang-Mills theory, J. High Energy Phys. 03 (2002) 018.

[3] S. Badger, H. Frellesvig, and Y. Zhang, A two-loop fivegluon helicity amplitude in QCD, J. High Energy Phys. 12 (2013) 045.

[4] S. Badger, G. Mogull, A. Ochirov, and D. O'Connell, A complete two-loop, five-gluon helicity amplitude in YangMills theory, J. High Energy Phys. 10 (2015) 064.

[5] T. Gehrmann, J. M. Henn, and N. A. Lo Presti, Analytic form of the Two-Loop Planar Five-Gluon All-Plus-Helicity
Amplitude in QCD, Phys. Rev. Lett. 116, 062001 (2016); Erratum, Phys. Rev. Lett. 116, 189903 (2016).

[6] D. C. Dunbar and W. B. Perkins, Two-loop five-point all plus helicity Yang-Mills amplitude, Phys. Rev. D 93, 085029 (2016).

[7] D. C. Dunbar, G. R. Jehu, and W. B. Perkins, Two-Loop Six Gluon all Plus Helicity Amplitude, Phys. Rev. Lett. 117, 061602 (2016).

[8] D. C. Dunbar, J. H. Godwin, G. R. Jehu, and W. B. Perkins, Analytic all-plus-helicity gluon amplitudes in QCD, Phys. Rev. D 96, 116013 (2017).

[9] S. Abreu, J. Dormans, F. Febres Cordero, H. Ita, B. Page, and V. Sotnikov, Analytic form of the planar two-loop fiveparton scattering amplitudes in QCD, J. High Energy Phys. 05 (2019) 084. 
[10] S. Badger, C. Brnnum-Hansen, H. B. Hartanto, and T. Peraro, Analytic helicity amplitudes for two-loop five-gluon scattering: The single-minus case, J. High Energy Phys. 01 (2019) 186.

[11] D. Chicherin, T. Gehrmann, J. M. Henn, P. Wasser, Y. Zhang, and S. Zoia, All Master Integrals for Three-Jet Production at Next-to-Next-to-Leading Order, Phys. Rev. Lett. 123, 041603 (2019).

[12] H. A. Chawdhry, M. A. Lim, and A. Mitov, Two-loop fivepoint massless QCD amplitudes within the integration-byparts approach, Phys. Rev. D 99, 076011 (2019).

[13] S. Badger, D. Chicherin, T. Gehrmann, G. Heinrich, J. M. Henn, T. Peraro, P. Wasser, Y. Zhang, and S. Zoia, Analytic form of the Full Two-Loop Five-Gluon All-Plus Helicity Amplitude, Phys. Rev. Lett. 123, 071601 (2019).

[14] S. G. Naculich, All-loop group-theory constraints for colorordered SU(N) gauge-theory amplitudes, Phys. Lett. B 707, 191 (2012).

[15] A.C. Edison and S. G. Naculich, SU(N) group-theory constraints on color-ordered five-point amplitudes at all loop orders, Nucl. Phys. B858, 488 (2012).

[16] S. D. Alston, D. C. Dunbar, and W. B. Perkins, Complex factorisation and recursion for one-loop amplitudes, Phys. Rev. D 86, 085022 (2012).

[17] V. Del Duca, L. J. Dixon, and F. Maltoni, New color decompositions for gauge amplitudes at tree and loop level, Nucl. Phys. B571, 51 (2000).

[18] Z. Bern and D. A. Kosower, Color decomposition of one loop amplitudes in gauge theories, Nucl. Phys. B362, 389 (1991).

[19] Z. Bern, G. Chalmers, L. J. Dixon, and D. A. Kosower, One Loop $N$ Gluon Amplitudes with Maximal Helicity Violation via Collinear Limits, Phys. Rev. Lett. 72, 2134 (1994).

[20] Z. Bern, L. J. Dixon, D. C. Dunbar, and D. A. Kosower, One loop selfdual and $N=4$ superYang-Mills, Phys. Lett. B 394, 105 (1997).

[21] Z. Bern, L. J. Dixon, and D. A. Kosower, On-shell recurrence relations for one-loop QCD amplitudes, Phys. Rev. D 71, 105013 (2005).

[22] Z. Bern, L. J. Dixon, and D. A. Kosower, One Loop Corrections to Five Gluon Amplitudes, Phys. Rev. Lett. 70, 2677 (1993).
[23] S. Catani, The Singular behavior of QCD amplitudes at two loop order, Phys. Lett. B 427, 161 (1998).

[24] Z. Kunszt, A. Signer, and Z. Trocsanyi, Singular terms of helicity amplitudes at one loop in QCD and the soft limit of the cross-sections of multiparton processes, Nucl. Phys. B420, 550 (1994).

[25] Z. Bern, L. J. Dixon, D. C. Dunbar, and D. A. Kosower, One loop $\mathrm{n}$ point gauge theory amplitudes, unitarity and collinear limits, Nucl. Phys. B425, 217 (1994).

[26] Z. Bern, L. J. Dixon, D. C. Dunbar, and D. A. Kosower, Fusing gauge theory tree amplitudes into loop amplitudes, Nucl. Phys. B435, 59 (1995).

[27] D. C. Dunbar, W. B. Perkins, and E. Warrick, The unitarity method using a canonical basis approach, J. High Energy Phys. 06 (2009) 056.

[28] D. C. Dunbar, G. R. Jehu, and W. B. Perkins, The two-loop n-point all-plus helicity amplitude, Phys. Rev. D 93, 125006 (2016).

[29] R. Britto, F. Cachazo, B. Feng, and E. Witten, Direct Proof of Tree-Level Recursion Relation in Yang-Mills Theory, Phys. Rev. Lett. 94, 181602 (2005).

[30] K. Risager, A Direct proof of the CSW rules, J. High Energy Phys. 12 (2005) 003.

[31] D. C. Dunbar, J. H. Ettle, and W. B. Perkins, Augmented recursion for one-loop gravity amplitudes, J. High Energy Phys. 06 (2010) 027.

[32] S. D. Alston, D. C. Dunbar, and W. B. Perkins, n-point amplitudes with a single negative-helicity graviton, Phys. Rev. D 92, 065024 (2015).

[33] D. C. Dunbar and W. B. Perkins, $\mathcal{N}=4$ supergravity nextto-maximally-helicity-violating six-point one-loop amplitude, Phys. Rev. D 94, 125027 (2016).

[34] C. Schwinn and S. Weinzierl, Scalar diagrammatic rules for Born amplitudes in QCD, J. High Energy Phys. 05 (2005) 006.

[35] D. A. Kosower, Light cone recurrence relations for QCD amplitudes, Nucl. Phys. B335, 23 (1990).

[36] Z. Bern, L. J. Dixon, and D. A. Kosower, A Two loop four gluon helicity amplitude in QCD, J. High Energy Phys. 01 (2000) 027. 